\section{INFLAMMATION \\ Myocardial infarct inflammation}

Nat. Med. 23, 1481-1487 (2017)

The molecular drivers of myocardial infarction (MI)-driven inflammation remain unclear. In Nature Medicine, King et al. show that uptake of cellular debris by cardiac macrophages after ischemic cell death activates the production of type I interferons. Activation of the transcription factor IRF3 and induction of the cytokineand chemokine-encoding genes Ifnbl and Cxcl10 are detected $4 \mathrm{~d}$ after MI in wild-type mice but not in mice deficient in the adaptor STING or the DNA sensor cGAS. Uptake of dying cardiomyocytes by $\mathrm{F} 4 / 80^{\text {hi }} \mathrm{Ly} 6 \mathrm{C}^{\text {lo }}$ phagocytic macrophages triggers the IRF3dependent recruitment of blood F4/80 10 Ly $6 \mathrm{C}^{\text {hi }}$ pro-inflammatory monocytes that amplify the inflammatory response. $\mathrm{Irf3}^{-1-}$ mice and mice deficient in the interferon receptor IFNAR are protected from death and show fewer signs of heart failure after MI. Treatment with IFNAR-neutralizing antibodies at 12 and $48 \mathrm{~h}$ after MI improves ventricular size, contractile function and survival in mice. $I V$

https://doi.org/10.1038/s41590-017-0037-3

\section{NEUROIMMUNOLOGY}

\section{Stress-induced depression}

Nat. Neurosci. 20, 1752-1760 (2017)

Exposure to chronic emotional stress is linked to the activation of innate immune responses and depression. In Nature Neuroscience, Menard et al. show that stressed mice develop increased permeability of the blood-brain barrier in the nucleus accumbens (NAc) region of the brain, a site associated with mood disorders. Stressed mice exhibit loss of the tight-junction protein claudin-5 and loss of blood-brain barrier integrity in the NAc but not in other brain regions. Loss of claudin- 5 expression is also observed in human subjects. Recruitment of circulating CCR2 $2^{+}$monocytes and accumulation of the cytokine IL- 6 in the NAc accompany reduced expression of claudin-5, all of which correlates with social avoidance and other measures of depression. These findings reveal how peripheral mediators contribute to depression.

https://doi.org/10.1038/s41590-017-0038-2

\section{ANTIMICROBIAL RESPONSE}

\section{Antibiotics-immune system interactions \\ Cell Host Microbe 22, 757-765 (2017)}

It is known that antibiotics not only target bacteria but also can alter host metabolism by damaging mitochondrial function. In Cell Host \& Microbe, Collins and co-workers investigate the effects of antibiotics on host metabolism and their influence on antibacterial responses. Using a mouse model of infection with Escherichia coli, they find that the production of gut epithelial metabolites is altered by treatment with antibiotics in a microbiota-independent manner. These antibiotics-triggered epithelial metabolites diminish the efficacy of antibiotics directed against pathogenic bacteria but can improve the phagocytic function of macrophages. In contrast, the application of antibiotics directly to macrophages impairs their phagocytic and killing function, probably through alterations in the mitochondriadependent respiratory burst. Treatment with antibiotics therefore exerts complex direct and indirect effects on host-cell metabolism, with effects on antimicrobial function.

https://doi.org/10.1038/s41590-017-0041-7

\section{NEUROIMMUNOLOGY \\ Macrophages: damage control}

J. Clin. Invest. https://doi.org/10.1172/JC190647 (2017)

Cranial irradiation as practised in cancer therapy is often associated with damage to the brain and results in neurological impairment. In the Journal of Clinical Investigation, Scadden and colleagues demonstrate that bone marrow-derived monocytesmacrophages are important for repairing such irradiation-induced brain injury. Using a mouse model of irradiation injury, the authors observe that these cells migrate to and are retained long term in the brain parenchyma. When mice are treated with the myeloid cell-stimulatory cytokine G-CSF, brain repair is improved in a manner dependent on bone marrow monocytesmacrophages. Cognitive function is similarly improved after treatment with G-CSF. This study demonstrates an unexpected connection between bone marrow-derived monocytes-macrophages and brain repair.

https://doi.org/10.1038/s41590-017-0040-8

\section{HUMORAL IMMUNITY \\ NKT cells aid antiviral responses}

Cell https://doi.org/10.1016/j.cell.2017.11.036

(14 December 2017)

Natural killer T cells (NKT cells) respond to glycolipid antigens presented on CD1 molecules and contribute to anti-bacterial humoral responses by providing cognate help to B cells. In Cell, Gaya et al. show that NKT cells also promote antibody responses during viral infection. Mice lacking CD1d develop fewer germinal centers and less virus-specific immunoglobulin G1 than do wild-type mice. NKT cell help is indirect and occurs by day 3 after infection, before the formation of germinal centers, at the periphery of $B$ cell follicles via their elaboration of IL-4. CD1d ${ }^{+} \mathrm{CD} 169^{+}$macrophages release the IL-18 that is needed for NKT cell activation and IL-4 production. This early wave of IL-4 released by NKT cells thereby enhances B cell antibody responses after viral infection.

https://doi.org/10.1038/s41590-017-0039-1 